\title{
Transformational leadership encourages residents' job crafting in surgical training: A mixed-methods study of residents' perceptions
}

LUIS CARLOS DOMINGUEZ ( $\sim$ carlosdot@unisabana.edu.co)

Universidad de La Sabana https://orcid.org/0000-0002-1595-8364

Diana Dolmans

Universiteit Maastricht Faculty of Health Medicine and Life Sciences

Willem de Grave

Universiteit Maastricht Faculty of Health Medicine and Life Sciences

Jeroen Donkers

Universiteit Maastricht Faculty of Health Medicine and Life Sciences

Alvaro Sanabria

Universidad de La Sabana

Laurents Stassen

Maastricht Universitair Medisch Centrum+

Research article

Keywords: job crafting, leadership, surgery, residency training, Colombia

Posted Date: August 12th, 2020

DOI: https://doi.org/10.21203/rs.3.rs-52900/v1

License: (1) This work is licensed under a Creative Commons Attribution 4.0 International License. Read Full License 


\section{Abstract}

Background Supervisors' leadership style can enhance resident performance, especially in terms of their ability to deal with the demands in the workplace and to take advantage of the available resources. Dealing with job demands and resources is known as job crafting, which has implications for the persistence of residents in training. The link between supervisors' leadership style and residents' job crafting, however, is not well understood.

Methods This mixed-methods study sought to explore the relationships between a transformational (team-oriented), transactional (taskoriented), and laissez-faire (passive) supervisory style and residents' job crafting and to explain these relationships. Residents filled out the Multifactor Leadership Questionnaire to rate their supervisors' leadership style and the Dutch job-crafting scale to assess their own job crafting. We tested the relationships using linear mixed effects regression analysis. To explain the ensuing results, we subsequently conducted a thematic analysis of semi-structured interviews with residents.

Results One hundred and sixteen residents from 7 surgical programs participated. A transformational leadership style had a positive effect on residents' job crafting $(b=0.19 ; 95 \% \mathrm{Cl} 0.08-0.32 ; p=0.009)$, whereas the transactional and laissez-faire styles did not. This could be explained by the fact that residents felt their transformational supervisors had a positive influence on the atmosphere for training and on the job resources available to them, and considered them positive role models for how to handle the demands of the environment.

Conclusion In residents' view, a transformational style is positively related to the ability to craft their jobs and therefore has implications for their persistence in training. Future research should explore supervisors' perspective on this relation and the effectiveness of leadership training for supervisors with a focus on resident outcomes, such as job crafting and persistence in training.

\section{Background}

The problem of residents dropping out of surgical training has received increasing attention because of its negative effects on the individual and on health systems. Residents' poor well-being in the workplace remains the main reason for the high dropout rate (1-4). Not only supervisors and program coordinators, but also residents are responsible for improving resident well-being: Supervisors play an important role in offering residents support to optimize their learning in the workplace. Residents, in their turn, must undertake action to optimize and gain control of the workplace for training $(5,6)$. Such actions are in keeping with the concept of job crafting (7). Successful job crafting may help explain why more than $80 \%$ of residents complete surgical training in different countries $(4,6,8,9)$.

Originating from the field of proactivity at work, job crafting explains how individuals are able to transform their job actively, instead of simply reacting passively to the working conditions to which they are exposed (10). According to job demands-resources (JD-R) theory, job crafting is exercised when a worker increases two groups of job resources: structural resources (those that foster autonomy) and social resources (those that improve relatedness, e.g., peer collaboration). Workers also craft their jobs by diminishing hindering job demands (e.g., conflicts) and by increasing challenging demands (those that stimulate personal growth and achievement, e.g., problem solving skills) (11). When workers craft their jobs, they can become more engaged, which is a positive well-being state as opposed to burnout. Work engagement, ultimately, is related to increased job crafting (12).

A preliminary study found that when residents' work engagement was high, their job crafting was negatively correlated with their intention to leave surgical training (5). A subsequent study offered insight into how residents craft their jobs to persist in training. They do so through the following six mechanisms: 1) building trust with supervisors, 2) being proactive in the workplace to gain responsibility, 3) seeking help from peers to deal with the demands of training, 4) seeing errors and frustrations as learning opportunities, 5) finding a suitable work-life balance, and 6) searching for challenging surgical tasks (13). In the said study, residents acknowledged that supervisors' leadership played an important role in the six mechanisms. Nonetheless, how supervisors' leadership drives residents' job crafting still represents a knowledge gap. There is a need for studies that explore how leaders can influence job crafting in different work contexts $(14,15)$. Such studies might help us better understand what actions leaders (i.e., supervisors) could take to support residents in the workplace.

Leaders make use of different styles to lead and motivate people. In the literature we find three leadership styles that prevail in organizations (14). The first is a transformational leadership style (TLS), which is team-oriented and aimed to raise awareness about collective interests in others (e.g., vision of the organization, high standards) (14). Those who have a TLS elicit awareness and knowledge of their own job in others and serve as role models (14-16); they are committed to the organizational culture and justice at 
work, diminish bullying and burnout, and foster work engagement $(14,15,17-20)$. Conversely, a transactional leadership style (TrLS) is task-oriented and aims to fulfill objectives, ensure standards and monitor outcomes (14). Those who have a TrLS offer rewards or punishments to others according to their performance during tasks. They are likely to discourage and decrease empowerment, job satisfaction, and work engagement (21-24). Finally, a laissez-faire leadership style (LfLS) is passive and aims to meet the expectations of the organization. Those who have a LfLS are rarely present and they diminish job satisfaction, productivity, and job effectiveness (14, 25).

In this study, we hypothesized that a TLS in supervisors would be positively associated with residents' job crafting in surgical training. We also hypothesized that a TrLS and an LfLS would be negatively associated with residents' job crafting, due to the aforementioned characteristics. The study addressed the following research question: To what extent are supervisors' leadership styles associated with residents' job crafting in surgical training and why, from residents' perspective?

\section{Methods}

We employed a sequential, explanatory mixed-methods research design (26). More specifically, we first measured supervisors' leadership style and residents' job crafting using questionnaires. Then, we conducted interviews with residents to gain a deeper understanding of why these styles were perceived to affect their proactive behaviors. Such a research approach responds to the need to integrate multiple sources of data that together, by drawing on the strengths of quantitative and qualitative methods, can help explain the complex relationship between leadership and job crafting (27-29). The Commission for Medical Education of the Universidad de la Sabana granted ethical approval.

Quantitative phase

\section{Setting and participants}

This study was conducted in Colombia where residents hold full-time positions in healthcare institutions during four years of surgical training. Annual tuition for training in private programs is close to 12,000 USD, which includes 66 hours of duty per week. Three out of 20 residencies in surgery are accredited as high-quality programs in accordance with national standards; two more are in the process of attaining that accreditation. Considering the number of affiliated institutions, each program hosts more than 30 surgical supervisors. Residents' burnout rate is $33 \%$ and almost $11.9 \%$ of residents have serious intentions to leave training (5).

We invited all the residents of seven surgical programs (a total of 136 residents) to participate voluntarily in the quantitative phase between October and December, 2018. We chose the five programs that were either accredited or in the process of obtaining high-quality accreditation. We included two others that had the longest standing tradition in the country. We emphasized confidentiality and anonymity of data.

\section{Measures}

Supervisors' leadership styles (independent variables)

The variables we measured were three: TLS, TrLS and LfLS. We obtained permission to administer the Multifactor Leadership Questionnaire (MLQ-5X) to residents to evaluate these styles in their supervisors (15). We asked residents to rate their supervisors in general, hence not their individual supervisors, direct supervisor, or the program director. We chose this approach because, first, residents interacted with multiple supervisors on a daily basis; second, the high number of supervisors per program limited the feasibility of each resident rating them all; and finally, program directors were not fully involved in clinical supervision. Residents were instructed to rate 'the standard surgeon who supervises residents (in the workplace) in the program that you are enrolled in...' on a 5-point scale $(1=$ not at all; 5 = frequently, if not always). This instruction followed the principle of standard reference by which decision-makers - residents in this case - assign intermediate values to their expectations, ranging from best to worst (30).

\section{Residents' job crafting (dependent variables)}

We measured five variables that corresponded to each job-crafting domain: (1) increasing structural resources; (2) increasing social resources; (3) decreasing hindering demands; (4) increasing challenging demands; and (5) global job crafting. Residents rated their job crafting on the published version of the Dutch job-crafting scale (DJCS) on a 5-point scale ( $1=$ never; $5=$ very often). Each of the four subscales had a Cronbach's a of $>0.70$ (7). 


\section{Statistical analysis}

We first calculated the descriptive statistics for all variables. For each program, we clustered the individual answers on the MLQ-5X and DJCS to represent the overall scores for each leadership style and job crafting. In these analyses, we calculated means, standard deviations (SD), and 95\% confidence intervals (Cl) for the independent and dependent variables per program. We conducted one-way univariate analyses of variance (ANOVAs) to identify significant differences in scores between programs. We adjusted p-values of ANOVAs (Bonferroni correction) considering the number of tests. We computed Cronbach's a for questionnaires.

We used R (R Core Team, 2019) and Ime4 (31) to perform a linear mixed effects analysis of the relationship between job crafting and leadership styles. As fixed effects, we entered the leadership styles into the model (without interaction term). As a random effect, we had intercepts for the program. We therefore applied the following mixed-effect model to the data in which JC represented global job crafting (or each one of the four job-crafting variables):

$\mathrm{JC} \sim \mathrm{TLS}+\mathrm{TrLS}+\mathrm{LfLS}+(1 \mid$ program $)$

Visual inspection of residual plots did not reveal any serious deviations from homoscedasticity or normality. P-values were obtained by likelihood ratio tests of the full model with the effect in question against the model without the effect. For each fixed effect we reported the $b$ estimate, the $95 \% \mathrm{Cl}$, and the chi-square ( $\mathrm{df}=1$ ) p-value (significance level of $<0.05$ ). In general, b estimates around $0.10,0.25$, and 0.40 can be interpreted as small, medium, and large effects (32). For the random effect we reported the variance, standard deviation $(\mathrm{SD})$, the $95 \% \mathrm{Cl}$, and a simulation-based p-value (significance level of $<0.05$ ). We also calculated the interclass correlation for the random effect $(I C C=$ variance $($ program $) /($ variance $($ program $)+$ variance $($ residuals $)))$. Finally, we assessed the model's goodness of fit with the R2m (marginal R-squared) and the R2c (conditional R-squared) indices (33).

Qualitative phase

The qualitative phase took place between January and April, 2019. First, we developed an interview guide to explore the quantitative results (Appendix 1). Then, LCD and AS recruited 20 residents using stratified sampling based on demographics (e.g., program and year of training). All interviews were conducted by phone. All participants gave verbal informed consent to be involved in the interviews after we had explained the mechanisms to ensure anonymity, confidentiality, and management of information. LCD conducted the individual, in-depth interviews in Spanish, using a non-technical language to guide the participants in each leadership style and formulate key questions. Upon completion, all interviews were immediately audiotaped and transcribed verbatim. Then, LCD and AS performed a thematic analysis of all transcriptions. With this method, we sought to identify themes within our dataset (34) and reach thematic saturation, that is, the stage in which no new categories appeared, previous data did not require any more modifications, and no additional data were needed (35). After 10 interviews, LCD and AS had identified the main themes, but felt more information was needed to explain some aspects of these themes in depth. We therefore conducted 14 interviews in total, after which LCD and AS felt thematic sufficiency was reached. All authors subsequently discussed these themes iteratively to reach consensus.

In this analysis, we acknowledged, through reflexivity, that we as researchers add meaning to the findings. The researchers have different backgrounds, experiences, and perspectives on leadership and residency training, which may have influenced data collection and analysis. LCD, AS, and LS are surgeons, supervisors, and directors of surgical programs. DD and WdG have extensive experience as educational researchers in workplace-based learning in residency training. Ultimately, our interpretation of the findings was influenced by the concepts of JD-R theory (11). Our different perspectives combined with the said theoretical concepts may benefit the strength of the study and the transferability of its findings. Finally, in all stages of the qualitative phase, we followed recommendations for the translation of information, in our case from Spanish into English language (36).

\section{Results}

Quantitative results

We included 116 residents from seven programs (92.6\% response rate). Table 1 presents the characteristics of the participants. The mean scores of the MLQ-5X were: TLS $=3.39 \pm 0.72 ;$ TrLS $=2.93 \pm 0.46$; and LfLS $=2.07 \pm 0.75$ ( $1-5$ range). The mean scores of the DJCS were: global job crafting $=3.50 \pm 0.41$; job crafting to increase 1 ) structural resources $=4.35 \pm 0.52 ; 2)$ social resources $=3.75 \pm$ 0.66 ; and 3 ) challenging demands $=3.50 \pm 0.61$ ( $1-5$ range); and job crafting to diminish hindering demands $=2.59 \pm 0.73$. Table 2 shows the results of the ANOVAs for the main variables between programs. After Bonferroni correction $(p<0.006)$, we identified 
significant differences in the scores for global job crafting to increase social resources and to increase challenging demands. Similarly, we identified significant differences in the scores for a TLS and a TrLS. The Cronbach's a of the MLQ-5X was 0.94. Cronbach's alphas for the subscales of the DJCS ranged from 0.63 to 0.78 .

Table 1

Descriptive characteristics of programs and participants

\begin{tabular}{|ll|}
\hline 1. Information on participants & 116 \\
\hline $\begin{array}{l}\text { Number of participants (global) } \\
\text { Age (mean, standard deviation, range) }\end{array}$ & Age: $28.59 \pm 2.48(22-$ \\
\hline $\begin{array}{l}\text { Male (number and percentage) } \\
\text { Age (mean, standard deviation, range) }\end{array}$ & $69(59.48 \%)$ \\
\hline Female (number and percentage) & Age: $28.81 \pm 2.77(22-$ \\
\hline Age (mean, standard deviation, range) & $36)$ \\
\hline Number and percentage of residents per year of training & $47(40.52 \%)$ \\
\hline Year 1 & Age: $28.27 \pm 2(24-34)$ \\
\hline Year 2 & $34(29.31 \%)$ \\
\hline Year 3 & $24(20.69 \%)$ \\
\hline Year 4 & $32(27.59 \%)$ \\
\hline 2. Information on programs & $26(22.41 \%)$ \\
\hline Number of programs & \\
\hline $\begin{array}{l}\text { Average number of residents per training program evaluating their supervisors' leadership styles } \\
\text { (range) }\end{array}$ & 7 \\
\hline
\end{tabular}


Table 2

Descriptive statistics and one-way Analyses of Variance (ANOVAs) of the relationship between residents' job crafting and supervisors' leadership style per program

\begin{tabular}{|c|c|c|c|c|c|c|c|c|c|c|}
\hline \multirow{2}{*}{$\begin{array}{l}\text { Variable } \\
\text { Global }(n=116)\end{array}$} & \multicolumn{8}{|c|}{ Residency program (number of residents per program) } & \multicolumn{2}{|c|}{$\begin{array}{l}\text { One-way } \\
\text { ANOVA }\end{array}$} \\
\hline & $\begin{array}{l}\text { Prog. } 1 \\
(n=21)\end{array}$ & $\begin{array}{l}\text { Prog. } 2 \\
(n=20)\end{array}$ & $\begin{array}{l}\text { Prog. } 3 \\
(n=16)\end{array}$ & $\begin{array}{l}\text { Prog. } 4 \\
(n=21)\end{array}$ & $\begin{array}{l}\text { Prog. } 5 \\
(n=16)\end{array}$ & $\begin{array}{l}\text { Prog. } 6 \\
(n=12)\end{array}$ & $\begin{array}{l}\text { Prog. } 7 \\
(n=10)\end{array}$ & $\mathrm{F}$ & \multicolumn{2}{|l|}{$\mathrm{p}^{\star \star}$} \\
\hline \multicolumn{11}{|c|}{ 1. Job crafting self-rated by residents on the Dutch job-crafting scale* (means, SD and $95 \% \mathrm{Cl}$ ) } \\
\hline Global & $\begin{array}{l}3.50 \pm \\
0.41 \\
(3.43- \\
3.58)\end{array}$ & $\begin{array}{l}3.30 \pm \\
0.32 \\
(3.10- \\
3.45)\end{array}$ & $\begin{array}{l}3.32 \pm \\
0.37 \\
(3.15- \\
3.50)\end{array}$ & $\begin{array}{l}3.42 \pm \\
0.22 \\
(3.30- \\
3.54)\end{array}$ & $\begin{array}{l}3.73 \pm \\
0.19 \\
(3.56- \\
3.90)\end{array}$ & $\begin{array}{l}3.47 \pm \\
0.30 \\
(3.31- \\
3.63)\end{array}$ & $\begin{array}{l}3.59 \pm \\
0.50 \\
(3.26- \\
3.91)\end{array}$ & $\begin{array}{l}3.88 \pm \\
0.53 \\
(3.49- \\
4.26)\end{array}$ & 4.98 & 0.0002 \\
\hline $\begin{array}{l}\text { Increasing } \\
\text { structural } \\
\text { resources }\end{array}$ & $\begin{array}{l}4.35 \pm \\
0.52 \\
(4.25- \\
4.45)\end{array}$ & $\begin{array}{l}4.25 \pm \\
0.46 \\
(4.04- \\
4.46)\end{array}$ & $\begin{array}{l}4.23 \pm \\
0.45 \\
(4.01- \\
4.41)\end{array}$ & $\begin{array}{l}4.31 \pm \\
0.33 \\
(4.13- \\
4.49)\end{array}$ & $\begin{array}{l}4.53 \pm \\
0.36 \\
(4.36- \\
4.69)\end{array}$ & $\begin{array}{l}4.27 \pm \\
0.43 \\
(4.04- \\
4.50)\end{array}$ & $\begin{array}{l}4.68 \pm \\
0.28 \\
(4.49- \\
4.86)\end{array}$ & $\begin{array}{l}4.22 \pm \\
1.19 \\
(3.36- \\
5.07)\end{array}$ & 1.75 & 0.11 \\
\hline $\begin{array}{l}\text { Increasing social } \\
\text { resources }\end{array}$ & $\begin{array}{l}3.75 \pm \\
0.66 \\
(3.63- \\
3.88)\end{array}$ & $\begin{array}{l}3.75 \pm \\
0.60 \\
(3.47- \\
4.02)\end{array}$ & $\begin{array}{l}3.35 \pm \\
0.65 \\
(3.04- \\
3.66)\end{array}$ & $\begin{array}{l}3.8 \pm \\
0.41 \\
(3.57- \\
4.02)\end{array}$ & $\begin{array}{l}4.01 \pm \\
0.57 \\
(3.75- \\
4.28)\end{array}$ & $\begin{array}{l}3.75 \pm \\
0.53 \\
(3.46- \\
4.03)\end{array}$ & $\begin{array}{l}3.40 \pm \\
0.80 \\
(2.89- \\
3.92)\end{array}$ & $\begin{array}{l}4.4 \pm \\
0.65 \\
(3.92- \\
4.87)\end{array}$ & 4.63 & 0.0003 \\
\hline $\begin{array}{l}\text { Decreasing } \\
\text { hindering } \\
\text { demands }\end{array}$ & $\begin{array}{l}2.59 \pm \\
0.73 \\
(2.46- \\
2.73)\end{array}$ & $\begin{array}{l}2.30 \pm \\
0.56 \\
(2.08- \\
2.59)\end{array}$ & $\begin{array}{l}2.59 \pm \\
0.72 \\
(2.25- \\
2.93)\end{array}$ & $\begin{array}{l}2.32 \pm \\
0.75 \\
(1.92- \\
2.72)\end{array}$ & $\begin{array}{l}2.93 \pm \\
0.84 \\
(2.55- \\
3.31)\end{array}$ & $\begin{array}{l}2.57 \pm \\
0.68 \\
(2.20- \\
2.93)\end{array}$ & $\begin{array}{l}2.58 \pm \\
0.72 \\
(2.12- \\
3.04)\end{array}$ & $\begin{array}{l}2.91 \pm \\
0.68 \\
(2.42- \\
3.40)\end{array}$ & 1.95 & 0.07 \\
\hline $\begin{array}{l}\text { Increasing } \\
\text { challenging } \\
\text { demands }\end{array}$ & $\begin{array}{l}3.50 \pm \\
0.61 \\
(3.39- \\
3.61)\end{array}$ & $\begin{array}{l}3.08 \pm \\
0.47 \\
(2.87- \\
3.30)\end{array}$ & $\begin{array}{l}3.28 \pm \\
0.40 \\
(3.09- \\
3.46)\end{array}$ & $\begin{array}{l}3.5 \pm \\
0.53 \\
(3.21- \\
3.78)\end{array}$ & $\begin{array}{l}3.61 \pm \\
0.62 \\
(3.33- \\
3.90)\end{array}$ & $\begin{array}{l}3.47 \pm \\
0.56 \\
(3.17- \\
3.77)\end{array}$ & $\begin{array}{l}3.88 \pm \\
0.76 \\
(3.39- \\
4.37)\end{array}$ & $\begin{array}{l}4.18 \pm \\
0.38 \\
(3.90- \\
4.45)\end{array}$ & 6.34 & 0.00001 \\
\hline \multicolumn{11}{|c|}{$\begin{array}{l}\text { 2. Supervisors' leadership style per program rated by residents on the Multifactor Leadership Questionnaire* (means, SD and } 95 \% \\
\text { Cl) }\end{array}$} \\
\hline Transformational & $\begin{array}{l}3.39 \pm \\
0.72 \\
(3.26- \\
3.53)\end{array}$ & $\begin{array}{l}3.10 \pm \\
0.58 \\
(2.84- \\
3.37)\end{array}$ & $\begin{array}{l}2.86 \pm \\
0.60 \\
(2.57- \\
3.14)\end{array}$ & $\begin{array}{l}3.01 \pm \\
0.66 \\
(2.65- \\
3.37)\end{array}$ & $\begin{array}{l}3.60 \pm \\
0.62 \\
(3.32- \\
3.89)\end{array}$ & $\begin{array}{l}3.87 \pm \\
0.61 \\
(3.54- \\
4.20)\end{array}$ & $\begin{array}{l}3.71 \pm \\
0.57 \\
(3.35- \\
4.07)\end{array}$ & $\begin{array}{l}4.09 \pm \\
0.46 \\
(3.76- \\
4.42)\end{array}$ & 9.39 & 0.00001 \\
\hline Transactional & $\begin{array}{l}2.93 \pm \\
0.46 \\
(2.85- \\
3.02)\end{array}$ & $\begin{array}{l}2.72 \pm \\
0.40 \\
(2.54- \\
2.91)\end{array}$ & $\begin{array}{l}2.84 \pm \\
0.45 \\
(2.63- \\
3.05)\end{array}$ & $\begin{array}{l}2.75 \pm \\
0.39 \\
(2.54- \\
2.96)\end{array}$ & $\begin{array}{l}2.99 \pm \\
0.46 \\
(2.78- \\
3.20)\end{array}$ & $\begin{array}{l}3.16 \pm \\
0.44 \\
(2.92- \\
3.40)\end{array}$ & $\begin{array}{l}2.93 \pm \\
0.36 \\
(2.70- \\
3.15)\end{array}$ & $\begin{array}{l}3.37 \pm \\
0.48 \\
(3.02- \\
3.71)\end{array}$ & 3.96 & 0.001 \\
\hline Laissez-faire & $\begin{array}{l}2.07 \pm \\
0.75 \\
(1.93- \\
2.21)\end{array}$ & $\begin{array}{l}2.04 \pm \\
0.87 \\
(1.64- \\
2.44)\end{array}$ & $\begin{array}{l}2.17 \pm \\
0.59 \\
(1.89- \\
2.45)\end{array}$ & $\begin{array}{l}1.95 \pm \\
0.70 \\
(1.57- \\
2.33)\end{array}$ & $\begin{array}{l}2.29 \pm \\
0.81 \\
(1.92- \\
2.66)\end{array}$ & $\begin{array}{l}1.89 \pm \\
0.90 \\
(1.40- \\
2.37)\end{array}$ & $\begin{array}{l}1.97 \pm \\
0.63 \\
(1.57- \\
2.38)\end{array}$ & $\begin{array}{l}2.07 \pm \\
0.67 \\
(1.59- \\
2.55)\end{array}$ & 0.61 & 0.71 \\
\hline \multicolumn{11}{|c|}{$\mathrm{SD}=$ standard deviation; $95 \% \mathrm{Cl}=95 \%$ confidence interval; Prog. = program; Prob. = probability $; \mathrm{F}=\mathrm{F}$ statistic } \\
\hline \multicolumn{11}{|l|}{ * (Range 1-5) } \\
\hline \multicolumn{11}{|c|}{ Degrees of freedom = 6 (in all cases) } \\
\hline **Probability wa & sted $t$ & .006 & ron & ion) & & & & & & \\
\hline
\end{tabular}

Table 3 presents the linear mixed effects analysis of the relationship between supervisors' leadership styles and global job crafting. A TLS had a positive effect on global job crafting $(b=0.19 ; 95 \% \mathrm{Cl} 0.08-0.32 ; p=0.009)$. In general, the difference between programs regarding the effect of leadership style on job crafting was relatively small (ICC ranged between 0.002 and 0.16 ). We found no significant relationships between a TrLS and LfLS and global job crafting. 
Table 3

Linear mixed effects analysis of the relationship between supervisors' leadership styles and residents' global job crafting

\begin{tabular}{|c|c|c|c|c|c|c|c|c|c|c|c|c|}
\hline \multirow{3}{*}{$\begin{array}{l}\text { Dependent } \\
\text { variable }\end{array}$} & \multicolumn{6}{|c|}{ Fixed effects } & \multirow{2}{*}{\multicolumn{3}{|c|}{$\begin{array}{l}\text { Random effects (adjusted per } \\
\text { program) }\end{array}$}} & \multirow[t]{3}{*}{ ICC } & \multirow{2}{*}{\multicolumn{2}{|c|}{$\begin{array}{l}\text { Goodness } \\
\text { of fit }\end{array}$}} \\
\hline & \multicolumn{2}{|c|}{$\begin{array}{l}\text { Transformational } \\
\text { style }\end{array}$} & \multicolumn{2}{|c|}{$\begin{array}{l}\text { Transactional } \\
\text { style }\end{array}$} & \multicolumn{2}{|c|}{$\begin{array}{l}\text { Laissez-faire } \\
\text { style }\end{array}$} & & & & & & \\
\hline & $\begin{array}{l}\mathrm{b}(95 \% \\
\mathrm{Cl})\end{array}$ & $p$ & $\begin{array}{l}\mathrm{b}(95 \% \\
\mathrm{Cl})\end{array}$ & $p$ & $\begin{array}{l}\mathrm{b}(95 \% \\
\mathrm{Cl})\end{array}$ & $p$ & $\begin{array}{l}\text { Variance } \\
\text { (SD, 95\% } \\
\text { Cl) }\end{array}$ & $\begin{array}{l}\text { Residual } \\
\text { (SD, 95\% } \\
\text { Cl) }\end{array}$ & $\mathrm{p}$ & & $\mathrm{R} 2 \mathrm{~m}$ & $\mathrm{R} 2 \mathrm{C}$ \\
\hline
\end{tabular}

\begin{tabular}{|c|c|c|c|c|c|c|c|c|c|c|c|c|}
\hline $\begin{array}{l}\text { Global job } \\
\text { crafting }\end{array}$ & $\begin{array}{l}0.19 \\
(0.08- \\
0.32)\end{array}$ & 0.009 & $\begin{array}{l}0.10 \\
(-0.06- \\
0.27)\end{array}$ & 0.21 & $\begin{array}{l}0.07 \\
(-0.01- \\
0.17)\end{array}$ & 0.10 & $\begin{array}{l}0.01 \pm \\
0.11 \\
(0.000- \\
0.24)\end{array}$ & $\begin{array}{l}0.12 \pm \\
0.34 \\
(0.30- \\
0.39)\end{array}$ & 0.02 & $10.23 \%$ & 0.18 & 0.26 \\
\hline \multicolumn{13}{|c|}{$\mathrm{b}=\mathrm{b}$ estimate; $\mathrm{SD}=$ standard deviation; $\mathrm{Cl}=$ confidence interval; $\mathrm{R} 2 \mathrm{~m}$ = marginal $\mathrm{R}$ squared; $\mathrm{R} 2 \mathrm{C}=$ conditional $\mathrm{R}$ squared } \\
\hline \multicolumn{13}{|c|}{ ICC $=$ interclass correlation for the random effect $($ ICC $=$ variance $($ program $) /($ variance $($ program $)+$ variance $($ residuals $)))$} \\
\hline
\end{tabular}

Table 4 shows the linear mixed effects analysis of the relationship between the leadership styles and each job-crafting domain. In general, a TLS was positively related to all job-crafting domains. In three domains (increasing structural and social resources and diminishing hindering demands), the effect was significant $(p<0.05)$. We found no significant effect between a TrLS and any jobcrafting domain. An LfLS had only a significant effect on job crafting to decrease hindering demands $(p=0.0001)$.

Table 4

Linear mixed effects analysis of the relationship between supervisors' leadership styles and four dimensions of residents' job crafting

\begin{tabular}{|c|c|c|c|c|c|c|c|c|c|c|c|c|}
\hline \multirow{3}{*}{$\begin{array}{l}\text { Job- } \\
\text { crafting } \\
\text { dimensions } \\
\text { (dependent } \\
\text { variables) }\end{array}$} & \multicolumn{6}{|c|}{ Fixed effects } & \multirow{2}{*}{\multicolumn{3}{|c|}{$\begin{array}{l}\text { Random effects (adjusted per } \\
\text { program) }\end{array}$}} & \multirow[t]{3}{*}{ ICC } & \multirow{2}{*}{\multicolumn{2}{|c|}{$\begin{array}{l}\text { Goodness } \\
\text { of fit }\end{array}$}} \\
\hline & \multicolumn{2}{|c|}{$\begin{array}{l}\text { Transformational } \\
\text { style }\end{array}$} & \multicolumn{2}{|c|}{$\begin{array}{l}\text { Transactional } \\
\text { style }\end{array}$} & \multicolumn{2}{|c|}{$\begin{array}{l}\text { Laissez-faire } \\
\text { style }\end{array}$} & & & & & & \\
\hline & $\begin{array}{l}\text { b }(95 \% \\
\mathrm{Cl})\end{array}$ & $p$ & $\begin{array}{l}\text { b }(95 \% \\
\text { Cl) }\end{array}$ & $p$ & $\begin{array}{l}\mathrm{b}(95 \% \\
\mathrm{Cl})\end{array}$ & $p$ & $\begin{array}{l}\text { Variance } \\
(\mathrm{SD}, 95 \% \\
\mathrm{CI})\end{array}$ & $\begin{array}{l}\text { Residual } \\
\text { (SD, } \\
95 \% \mathrm{Cl})\end{array}$ & $p$ & & R2m & R2C \\
\hline $\begin{array}{l}\text { Increasing } \\
\text { structural } \\
\text { resources }\end{array}$ & $\begin{array}{l}0.16 \\
(0.04- \\
0.29)\end{array}$ & 0.009 & $\begin{array}{l}0.06 \\
(-0.12- \\
0.24)\end{array}$ & 0.50 & $\begin{array}{l}-0.10 \\
(-0.20- \\
0.005)\end{array}$ & 0.06 & $\begin{array}{l}0.01 \pm \\
0.11 \\
(0.000- \\
0.23)\end{array}$ & $\begin{array}{l}0.14 \pm \\
0.37 \\
(0.32- \\
0.43)\end{array}$ & 0.04 & $8.22 \%$ & 0.15 & 0.22 \\
\hline $\begin{array}{l}\text { Increasing } \\
\text { social } \\
\text { resources }\end{array}$ & $\begin{array}{l}0.20 \\
(0.005- \\
0.40)\end{array}$ & 0.04 & $\begin{array}{l}0.19 \\
(-0.09- \\
0.48)\end{array}$ & 0.18 & $\begin{array}{l}-0.05 \\
(-0.22- \\
0.11)\end{array}$ & 0.51 & $\begin{array}{l}0.05 \pm \\
0.22 \\
(0.03- \\
0.45)\end{array}$ & $\begin{array}{l}0.35 \pm \\
0.59 \\
(0.51- \\
0.67)\end{array}$ & 0.007 & $12.88 \%$ & 0.09 & 0.21 \\
\hline $\begin{array}{l}\text { Decreasing } \\
\text { hindering } \\
\text { demands }\end{array}$ & $\begin{array}{l}0.27 \\
(0.06- \\
0.47)\end{array}$ & 0.01 & $\begin{array}{l}-0.02 \\
(-0.34- \\
0.29)\end{array}$ & 0.88 & $\begin{array}{l}0.37 \\
(0.19- \\
0.56)\end{array}$ & 0.0001 & $\begin{array}{l}0.001 \pm \\
0.03 \\
(0.000- \\
0.23)\end{array}$ & $\begin{array}{l}0.46 \pm \\
0.68 \\
(0.59- \\
0.76)\end{array}$ & 0.39 & $0.21 \%$ & 0.16 & 0.16 \\
\hline $\begin{array}{l}\text { Increasing } \\
\text { challenging } \\
\text { demands }\end{array}$ & $\begin{array}{l}0.15 \\
(-0.02- \\
0.34)\end{array}$ & 0.09 & $\begin{array}{l}0.22 \\
(-0.02- \\
0.49)\end{array}$ & 0.08 & $\begin{array}{l}0.06 \\
(-0.08- \\
0.21)\end{array}$ & 0.39 & $\begin{array}{l}0.05 \\
(0.04- \\
0.45)\end{array}$ & $\begin{array}{l}0.29 \pm \\
0.53 \\
(0.46- \\
0.61)\end{array}$ & 0.004 & $15.79 \%$ & 0.09 & 0.23 \\
\hline \multicolumn{13}{|c|}{$\mathrm{b}=\mathrm{b}$ estimate; $\mathrm{SD}=$ standard deviation; $\mathrm{Cl}=$ confidence interval; $\mathrm{R} 2 \mathrm{~m}=$ marginal $\mathrm{R}$ squared; $\mathrm{R} 2 \mathrm{C}=$ conditional $\mathrm{R}$ squared } \\
\hline \multicolumn{13}{|c|}{ ICC $=$ interclass correlation for the random effect $($ ICC $=$ variance $($ program $) /($ variance $($ program $)+$ variance $($ residuals $)))$} \\
\hline \multicolumn{13}{|c|}{ p value: significance level of $<0.05$} \\
\hline
\end{tabular}

The marginal R2m was relatively low in all models, suggesting that factors other than supervisors' leadership styles may have influenced residents' job crafting. The conditional R2c was larger in all models, indicating that the effect of the program, albeit small, 
was important (Tables 3 and 4).

Qualitative results: Exploring the associations between supervisors' leadership style and residents' job crafting

Six participants were female (42.8\%). The distribution of participants by year of training was as follows: Year $1(n=4)$, year $2(n=3)$, year $3(n=3)$, and year $4(n=4)$. Three predominant themes emerged from the interviews. Table 5 gives an overview of representative quotations. 
Table 5

Quotes from the interviews illustrating the main themes

\section{Supervisors' leadership style \\ Theme 1: Supervisors' leadership style influences the atmosphere for training \\ Theme 2: Supervisors' leadership style influences the availability of job resources}

Transformational These supervisors, undoubtedly, create a better work environment. The hardest part of residency is not the workload, fasting, or the lack of sleep, but having a good relationship with the supervisors and peers. A quiet environment makes it easier for residents to work hard.

(Interview \#12: 4th -year male resident)
This type of leader offers the resident opportunities to be autonomous -within the framework of patient safety- to identify what [the resident] is doing well, and to have confidence ... They [the supervisors] tell him/her: "I want to operate on this patient with you," or "you are going to operate on this patient." These are ways to strengthen autonomy, which causes the resident to fight his/her own demons [fears].

(Interview \#3: 3rd-year male resident)

"Receiving positive influences makes you feel more motivated about what you do ... if you are in a positive atmosphere you will find much more enjoyment and love for what you do."

(Interview \#8: 4th-year male resident)
"When you are in contact with supervisors who provide good feedback and assessment, my mistakes can improve. Otherwise, the resident is left without knowing if he/she did things right or wrong." (Interview \#4: 3rd -year female resident)

\section{Theme 3: Supervisors' leadership style serves as role model for how to handle the environment}

All of us [residents] have difficult times during residency. If you have a role model, a supervisor with whom to share the anguish, that's good, that can help you to stay in the program. You admire some supervisors, not only because of their surgical capabilities, but also because of their integrity, because they are good human beings ... Sometimes you have big problems, but they can help you to see them smaller. These models make you see the problems from other angles to solve them.

(Interview \#1: 2nd -year female resident)

When we [residents] find a person who is charismatic, who salutes, who is decent, who has manners, who gets along well with all people, and who has good results and relationships with patients, we identify with that type of leader. A leader is someone who not only knows how to manage people, but also shows other people how to solve problems. Almost always, this type of leader has the solution to problems, easily and without collateral results.

(Interview \#14: 1st -year male resident)

"In an environment of trust the resident has a better development, while he/she loses his/her fears of being judged by his/her opinion and decisions ... You feel a safe environment where you will not be judged negatively."

(Interview \#8: 4th -year male resident)
"This supervisor supports good decisions [by residents], or appropriately corrects wrong decisions. This generates a good, safe, and positive environment, which leads to more calmness for the resident. One works better and has better results."

\section{(Interview \#9: 3rd -year male} resident)

"These supervisors are focused When leadership is based on punishment, the academic and work environments are hostile. Residents' behavior is based on fear. This is inappropriate practice [professional practice] for the patient. The resident is thinking how to avoid errors, to prevent a negative response from the supervisor. This generates greater stress for the resident ... The resident acts defensively and not proactively in the patient's favor. (Interview \#4: 3rd -year female resident) on the error and do not give feedback to the resident ... so the resident keeps making mistakes. That is not a good way to teach."

(Interview \#6: 4th -year male resident)
"A resident who has a good model someone who inspires him/her more, [expresses] confidence and encourages his/her improvement- is a better example than the one who is just waiting for an excuse to punish."

(Interview \#12: 4th -year male resident)

"They are people [supervisors] who were in a residency program in which their supervisors did the same [punishment]. They think that if there is no punishment, or a threat, residents will not perform well."

(Interview \#13: 4th -year female resident) 


\begin{tabular}{|ll|}
\hline $\begin{array}{l}\text { Supervisors' } \\
\text { leadership style }\end{array}$ & $\begin{array}{l}\text { Theme 1: Supervisors' leadership } \\
\text { style influences the atmosphere } \\
\text { for training }\end{array}$ \\
\hline
\end{tabular}

"Fear causes a lack of control over situations leading to wrong decisions. When one is afraid, one does not work well. That kind of supervisor generates fear." (Interview \#9: 3rd -year male resident)

\section{Theme 2: Supervisors' leadership style influences the availability of job resources}

When the resident makes a mistake, he/she loses his/her reward, that is: not getting to operate on patients. Then, he/she avoids mistakes because he/she will lose his/her reward. There is nothing more serious in education than to be afraid of making mistakes, because that is not the way a human being learns. If I make a mistake, they [supervisors] take away my opportunity to be in surgery! (Interview \#3: 3rd -year male resident)

"If a person is repeatedly punished and does not receive an adequate way to control the environment [from supervisors], he/she can fall into eternal punishment ... creating a poor work environment..."

(Interview \#14: 1st-year male resident)
"Supervisors know that the biggest punishment is to not allow residents to operate on patients, which discourages and demotivates them ... Then, a resident in an environment with a high workload and stress will not have good results."

(Interview \#12: 4th -year male resident)

Theme 3: Supervisors' leadership style serves as role model for how to handle the environment

A resident who faces punishment and reward models is uneasy about and dissatisfied with training. Some resilient people may not leave the program (perhaps the majority of residents), but there are others who do not endure that ... They do not sacrifice their calmness and happiness and they leave the program.

(Interview \#8: 4th -year male resident)

I do not want to be like that person [the supervisor]. I do not want that life for me. If you see that your supervisor is rude, bad-tempered, someone who is not able to control his/her anger (such people almost always have personal problems, divorces, and do not spend enough time at home), the resident may say: "this is not the life that I want in the future!" So, the resident leaves the program.

(Interview \#3: 3rd -year male resident)

Laissez-faire "These supervisors do not generate fear, but you do not feel supported, which affects the control of the environment."

(Interview \#9: 3rd -year male resident)

"Passivity creates a boring work environment."

(Interview \#2: 1st -year female resident)
"Those supervisors leave the resident alone with the problems ... They do not respond when they are needed." (Interview \#1: 2nd -year female resident)
"They are not a good model ... If I do not see a desire for leadership in the supervisor, he/she will not be a role model. [Their supervision] does not generate anything in me.

(Interview \#1: 2nd-year female resident)

If you have a disinterested, a passive supervisor who does the minimum required to fulfill, you are getting a bad model. Very little profit is taken from them, because they do not commit, do not make decisions and do not take appropriate care of situations ... They simply expend the least effort possible.

(Interview \#12: 4th -year male resident)

(Interview \#13: 4th -year female resident)

Many supervisors are passive leaders, many of them spend time with residents because it is their obligation. Although they are part of a surgical team, they are not real supervisors, they are not interested [in residents' education] ... They are indolent and indifferent to the resident ..., so there is no real connection between supervisor and resident ... It is not an enriching environment for learning.

(Interview \#11: 2nd -year female resident)
These supervisors do not ask me anything [clinical or academic issues], they do not contribute to my training, they are simply focused on their administrative duties ... this makes me feel that I'm wasting my time. They do not demand anything from me.

(Interview \#5: 1st-year female resident)
"That supervisor is not a good role model because he/she is an indecisive person, someone who confuses."

(Interview \#14: 1st -year male resident) 
Theme 1: Supervisors' leadership style influences the atmosphere for training in positive or negative ways

Residents mentioned that supervisors who had a TLS could promote a positive atmosphere for training and showed high standards of patient care. The personal strengths they attributed to such supervisors were altruism, integrity, resilience, and trustworthiness.

Residents valued this atmosphere because it made them feel free to discuss their fears and expectations of training, strengthening both their performance (e.g., decision-making and problem-solving skills) and readiness for practice. In residents' view, these supervisors stimulated them to stay in the program and pursue their training.

Conversely, residents characterized supervisors who had a TrLS as people who actively searched for errors, and were punitive and authoritative, which did not encourage them to take the lead in their own training on the job. In most cases, these supervisors created a hostile atmosphere for training where residents experienced fear and mistreatment, leading to defensive behaviors to hide errors and avoid punishment, and to more intentions to leave training.

Finally, residents mentioned that supervisors who had an LfLS showed a lack of commitment to patient care and residents' education and were perceived to help create a negative atmosphere in the workplace. Moreover, they contributed to more demands for the resident (i.e., more workload), resulting in unsafe care for patients. At the same time, however, the rare presence of these supervisors in the workplace encouraged residents to take more care of patients and to deal with workload and pressure.

Theme 2: Supervisors' leadership style influences the availability of job resources

Residents mentioned that supervisors who had a TLS offered the resident more job resources and challenges in the workplace (e.g., in the form of support, teaching, and feedback). Supervisors who had a TrLS and LfLS, by contrast, provided fewer of these resources and challenges, while creating more hindering demands (e.g., workload and pressure). For instance, supervisors who had a TrLS gave poor feedback and instruction and frequently punished residents by limiting opportunities to participate in surgical care and to take on new challenges (such as the opportunity to operate complex patients). In residents' views, these negative aspects of training led to psychological distress, a lack of autonomy, and more intentions to leave training.

Theme 3: Supervisors' leadership style serves as role model for how to handle the demands in the workplace

According to residents, supervisors with a TLS were positive role models. More specifically, these supervisors were capable of handling the work environment and finding solutions to difficult situations effectively, for instance when surgical complications or conflicts arose at work. Supervisors with a TrLS or an LfLS, on the other hand, were perceived as negative role models, because they created more demands for the resident (in the form of conflicts, ambiguity), while wielding ineffective strategies to solve difficult situations.

\section{Discussion}

The results from our survey demonstrated that supervisors' leadership styles and residents' job crafting differed significantly across the programs under scrutiny. Globally, however, we found the transformational leadership style to have a significant effect on residents' job crafting in both fixed and random effect analyses (with the interaction of programs). Residents valued supervisors with such a style for their positive influence on the training atmosphere and on the availability of job resources, and because they served as positive role models. Conversely, neither the transactional nor the laissez-faire style was found to have a significant effect on residents' job crafting. During the interviews, however, residents argued that these supervisors had a negative influence on the training atmosphere, the availability of job resources, and on role modeling.

We must view these results in relation to the existing research. Our findings echo those of previous research pointing to the positive influence of supervisors with a TLS on the atmosphere in the workplace $(20,37,38)$. In our study, a safe atmosphere - understood as a non-punitive and open environment for training - helped create favorable conditions for residents (e.g., trust in the supervisor, less power distance and less fear to discuss expectations), inducing them to search for more opportunities to participate in decision-making, to solve complex problems, and to cope with adversity. In other words, a positive atmosphere is one of the centerpieces of residents' job crafting. Our results, moreover, suggest that such a positive atmosphere may depend on supervisors' ability to create a deep connection with residents, which supervisors with a TLS do. These findings tie in nicely with previous studies on the importance of supervisors' behaviors to a positive learning climate and residents' well-being (39-41).

To our knowledge, this study is the first to explore the impact of a TLS in surgical education, as most studies have hitherto focused on its effects on clinical outcomes (patient safety and team performance) $(42,43)$. Moreover, our study emphasizes the importance of a 
TLS to a crucial aspect of residents' education, that is, residents' job crafting, considering the complexity of the surgical work environment for training. Our results also suggest that supervisors must not only offer residents structural resources (e.g., autonomy and responsibility), social resources (e.g., feedback and coaching), and more challenging demands (e.g., participation in complex cases), they must also encourage them to seek these, so that residents can craft their jobs efficiently and improve their performance. These results are in keeping with studies into the effect of a TLS on empowerment and autonomy in healthcare contexts $(22,44)$. We found that supervisors who embrace a TLS can help residents to gain control at work, by demonstrating effective ways to handle hindering demands and stressors (e.g., conflicts, frustrations of training). Few studies have considered judging surgeons who serve as role models by abilities other than their "surgical skills" and "mastery of technique." The ability to deal with conflicting demands and to cope with adversity are examples of what residents expect to learn from their supervisors beyond the traditional dexterity competences (45-47). Our findings indicate that supervisors should be aware of their modeling function with respect to these non-technical competences.

Our qualitative findings, on the other hand, suggested that supervisors with a TrLS have a negative influence on the atmosphere for training. Fear and power distance were important factors that explained such a hostile atmosphere. These factors, in turn, serve to illustrate how the supervisors who embrace this leadership style are disconnected from residents in the workplace: Ultimately, these supervisors negatively affect the availability of job resources and increase hindering demands (i.e., workload). Other studies have reported similar findings with respect to an authoritative leadership style in the supervisors $(20,24,37,38,48)$. Nonetheless, contrary to what we expected, we identified a positive association between an LfLS and residents' job crafting to diminish hindering demands. Hypothetically, this could be explained by the fact that residents were forced to take control of patient care and deal with clinical workload in face of poor supervision from the surgeons in charge. These observations deserve further investigation.

We acknowledge that the study has both strengths and limitations. A strength is that it adds information to the available evidence (conducted in non-healthcare settings from non-educational perspectives) supporting the positive relationship between transformational leadership and job crafting $(28,49,50)$. Moreover, adding a qualitative stage provided more depth and was useful since few studies have focused on the qualitative dimensions of a TLS $(23,51)$. A first limitation is that the data we collected only represented residents' perspective and, consequently, the study lacks a supervisor perspective. Secondly, we did not study the role of moderators in the relationship between supervisors' leadership and job crafting. Possible moderators are the organizational culture at the level of departments and institutions, as well as residents' attributes (e.g., self-efficacy and grit).

This study has implications for practice and research. It is essential that supervisors become transformational leaders, as it will help residents to become skilled job crafters. As suggested by our results, a TLS in the surgical context typifies supervisors who reveal welldeveloped personal strengths (e.g., integrity and trustworthiness) and commitment to high standards of patient care. They contribute to a positive atmosphere for training in which they offer residents support aimed to strengthen their performance, motivation, and readiness for practice. Finally, they serve as role models for residents, by demonstrating effective behaviors for handling the demands of the work environment. We believe that organizations committed to strengthening a healthy workforce should implement formal training not only in this type of leadership for supervisors, but also in bottom-up strategies for residents to teach them how to optimize job demands and resources (e.g., job-crafting training as part of residency training) $(12,20)$. Moreover, investing in transformational leadership development for supervisors could help strengthen residents' job fit in surgical training and reduce burnout and dropout, as has been identified in other work contexts $(38,52)$. Considering the limitations, we call for studies into the organizational influences (e.g., culture and power) on the relationship between supervisors' leadership and residents' job crafting. Moreover, supervisors' perspectives on this topic deserve investigation. Similarly, we invite future studies to explore the effect of transformational leadership training and development for supervisors on residents' job crafting.

\section{Conclusion}

In conclusion, a TLS of supervisors in surgery is related to the extent to which residents are able to optimize the job demands and resources for training in order to gain control of the work environment. This relationship is rooted in the positive influence of that leadership style on the environment for training, on role modeling, and on resources for the resident.

\section{Abbreviations}

\section{TLS}

transformational leadership style 
TrLS

transactional leadership style

LfLS

laissez-faire leadership style

\section{Declarations}

\section{Authors' contributions:}

L.C.D. was responsible for the conception of the study, collection and analysis of data, funding acquisition, investigation, methodology, project administration, software, writing of the original manuscript, and for the revision and editing of the final version.

D.D. contributed to the conception of the study, analysis of data, investigation, supervision, methodology, writing of the original manuscript, and to the revision and editing of the final version.

W.d.G. contributed to the conception of the study, analysis of data, investigation, methodology, supervision, writing of the original manuscript, and to the revision and editing of the final version.

J.D. contributed to the analysis of data, methodology, writing of the original manuscript, and to the revision and editing of the final version.

A.S. contributed to the conception of the study, collection and analysis of data, funding acquisition, investigation, methodology, supervision, writing of the original manuscript, and to the revision and editing of the final version.

L.S. contributed to the conception of the study, analysis of data, investigation, methodology, supervision, writing of the original manuscript, and to the revision and editing of the final version.

All authors have read and approved the manuscript

Competing interests: The authors declare that they have no competing interests

Funding: Universidad de la Sabana (Colombia). The funder had no role in the study design, data collection, analysis, interpretation of data or in writing the manuscript.

Availability of data and materials: We as authors declare our commitment to provide all data of the present study by request. 
Ethics Approval and consent for publication: The Commission of medical Education, Faculty of Medicine, Universidad de la Sabana (Colombia), granted the ethical approval.

During the quantitative stage, we explained the mechanisms to ensure anonymity, confidentiality, and management of information. All participants gave written consent to participate.

During the qualitative stage, all participants gave verbal informed consent, since interviews were conducted by phone, after we had explained the mechanisms to ensure anonymity, confidentiality, and management of information.

Consent for publication: Not applicable.

Acknowledgements: We would like to thank to all the residents that kindly accepted to participate in this study, and to Angelique van den Heuvel for the English language edition to the manuscript.

\section{References}

1. Sullivan MC, Yeo H, Roman SA, Ciarleglio MM, Cong X, Bell RH, et al. Surgical residency and attrition: Defining the individual and programmatic factors predictive of trainee losses. J Am Coll Surg [Internet]. 2013 Mar [cited 2018 May 25];216(3):461-71. Available from: http://linkinghub.elsevier.com/retrieve/pii/S1072751512013403.

2. Bongiovanni T, Yeo H, Sosa JA, Yoo PS, Long T, Rosenthal M, et al. Attrition from surgical residency training: Perspectives from those who left. Am J Surg [Internet]. 2015 Oct [cited 2018 May 25];210(4):648-54. Available from: http://linkinghub.elsevier.com/retrieve/pii/S0002961015003657.

3. Adams S, Ginther DN, Neuls E, Hayes P. Attitudes and factors contributing to attrition in Canadian surgical specialty residency programs. Can J Surg [Internet]. 2017 Aug 1 [cited 2018 May 25];60(4):247-52. Available from: http://canjsurg.ca/vol60-issue4/604-247/.

4. Khoushhal Z, Hussain MA, Greco E, Mamdani M, Verma S, Rotstein O, et al. Prevalence and causes of attrition among surgical residents a systematic review and meta-analysis. In: JAMA Surgery [Internet]. 2017 [cited 2018 May 25]. p. 265-72. Available from: http://archsurg.jamanetwork.com/article.aspx?doi=10.1001/jamasurg.2016.4086.

5. Dominguez LC, Stassen L, de Grave W, Sanabria A, Alfonso E, Dolmans D. Taking control: Is job crafting related to the intention to leave surgical training? Koniaris LG, editor. PLoS One [Internet]. 2018 Jun 1 [cited 2018 Jun 2];13(6):e0197276. Available from: http://www.ncbi.nlm.nih.gov/pubmed/29856750.

6. Forel D, Vandepeer M, Duncan J, Tivey DR, Tobin SA. Leaving surgical training: some of the reasons are in surgery. ANZ J Surg [Internet]. 2018 May 1 [cited 2020 Mar 21];88(5):402-7. Available from: http://www.ncbi.nlm.nih.gov/pubmed/29411485.

7. Bakker AB, Ficapal-Cusí P, Torrent-Sellens J, Boada-Grau J, Hontangas-Beltrán P. The Spanish version of the Job Crafting Scale. Psicothema [Internet]. 2018 Feb [cited 2018 Sep 6];30(1):136-42. Available from: http://www.ncbi.nlm.nih.gov/pubmed/29363483.

8. http://doi.wiley.com/10.1002/bjs.10926

Halim UA, Riding DM. Systematic review of the prevalence, impact and mitigating strategies for bullying, undermining behaviour and harassment in the surgical workplace. Br J Surg [Internet]. 2018 Oct 1 [cited 2020 Mar 21];105(11):1390-7. Available from: http://doi.wiley.com/10.1002/bjs.10926.

9. Bustraan J, Dijkhuizen K, Velthuis S, Van Der Post R, Driessen E, Van Lith JMM, et al. Why do trainees leave hospital-based specialty training? A nationwide survey study investigating factors involved in attrition and subsequent career choices in the Netherlands. Vol. 9, BMJ Open. BMJ Publishing Group; 2019.

10. Demerouti E, Peeters MCW, van den Heuvel M. Job Crafting Interventions: Do They Work and Why? In: Positive Psychological Intervention Design and Protocols for Multi-Cultural Contexts. Springer International Publishing; 2019. p. 103-25. 
11. Bakker AB, Demerouti E, Sanz-Vergel Al. Burnout and Work Engagement: The JD-R Approach. Annu Rev Organ Psychol Organ Behav [Internet]. 2014 [cited 2018 May 25];1(1):389-411. Available from:

https://www.isonderhouden.nl/doc/pdf/arnoldbakker/articles/articles_arnold_bakker_348.pdf.

12. Bakker AB. Strategic and proactive approaches to work engagement. Vol. 46, Organizational Dynamics. 2017.

13. Dominguez LC, Dolmans D, de Grave W, Sanabria A, Stassen LP. Job Crafting to Persist in Surgical Training: A Qualitative Study From the Resident's Perspective. J Surg Res [Internet]. 2019 Jul 4 [cited 2019 Mar 29];239:180-90. Available from: http://www.ncbi.nlm.nih.gov/pubmed/30844632.

14. Avolio BJ. Full Range Leadership Development. Second Edi. United States of America: SAGE Publications; 2013.280 p.

15. Antonakis J, Avolio BJ, Sivasubramaniam N. Context. and leadership: an examination of the nine-factor full-range leadership theory using the Multifactor Leadership Questionnaire. Leadersh Q [Internet]. 2003 Jun 1 [cited 2018 Dec 21];14(3):261-95. Available from: https://www.sciencedirect.com/science/article/pii/S1048984303000304.

16. Lievens I, Vlerick P. Transformational leadership and safety performance among nurses: the mediating role of knowledge-related job characteristics. J Adv Nurs [Internet]. 2014 Mar [cited 2019 Apr 3];70(3):651-61. Available from: http://www.ncbi.nlm.nih.gov/pubmed/23981245.

17. Gillet N, Fouquereau E, Bonnaud-Antignac A, Mokounkolo R, Colombat P. The mediating role of organizational justice in the relationship between transformational leadership and nurses' quality of work life: A cross-sectional questionnaire survey. Int $J$ Nurs Stud [Internet]. 2013 Oct [cited 2019 Apr 3];50(10):1359-67. Available from: http://www.ncbi.nlm.nih.gov/pubmed/23298792.

18. Mills CB, Keller M, Chilcutt A, Nelson MD. No Laughing Matter: Workplace Bullying, Humor Orientation, and Leadership Styles. Workplace Health Saf [Internet]. 2019 Apr 21 [cited 2019 Apr 3];67(4):159-67. Available from: http://www.ncbi.nlm.nih.gov/pubmed/30574845.

19. Arnold KA. Transformational leadership and employee psychological well-being: A review and directions for future research. J Occup Health Psychol [Internet]. 2017 Jul [cited 2019 Apr 3];22(3):381-93. Available from: http://doi.apa.org/getdoi.cfm? doi $=10.1037 /$ ocp0000062.

20. 10.3389/fpsyg.2018.02784/full

Mazzetti G, Vignoli M, Petruzziello G, Palareti L. The Hardier You Are, the Healthier You Become. May Hardiness and Engagement Explain the Relationship Between Leadership and Employees' Health? Front Psychol [Internet]. 2019 Jan 14 [cited 2019 Apr 3];9. Available from: https://www.frontiersin.org/article/10.3389/fpsyg.2018.02784/full.

21. Tsuno K, Norito K. Multifactor leadership styles and new exposure to workplace bullying: a six-month prospective study. Ind Health [Internet]. 2015 [cited 2019 Apr 3];53(2):139-51. Available from: http://www.ncbi.nlm.nih.gov/pubmed/25382384.

22. Khan BP, Quinn Griffin MT, Fitzpatrick JJ. Staff Nurses' Perceptions of Their Nurse Managers' Transformational Leadership Behaviors and Their Own Structural Empowerment. JONA J Nurs Adm [Internet]. 2018 Dec [cited 2019 Apr 3];48(12):609-14. Available from: http://www.ncbi.nlm.nih.gov/pubmed/30407928.

23. Morsiani G, Bagnasco A, Sasso L. How staff nurses perceive the impact of nurse managers' leadership style in terms of job satisfaction: a mixed method study. J Nurs Manag [Internet]. 2017 Mar [cited 2019 Apr 3];25(2):119-28. Available from: http://www.ncbi.nlm.nih.gov/pubmed/27917561.

24. The Influence of Nurse Manager Leadership Style on Staff Nurse Work Engagement Manning J. The Influence of Nurse Manager Leadership Style on Staff Nurse Work Engagement. JONA J Nurs Adm [Internet]. 2016 Sep [cited 2019 Apr 3];46(9):438-43. Available from: http://www.ncbi.nlm.nih.gov/pubmed/27496584.

25. Skogstad A, Einarsen S, Torsheim T, Aasland MS, Hetland H. The destructiveness of laissez-faire leadership behavior. J Occup Health Psychol [Internet]. 2007 Jan [cited 2019 May 8];12(1):80-92. Available from: http://doi.apa.org/getdoi.cfm? doi=10.1037/1076-8998.12.1.80.

26. Creswell JW, Guetterman TC. Mixed Methods Design. In: Educational research: planning, conducting, and evaluating quantitative and qualitative research. 6th editon. Creswell JW, Guetterman TC, editors. UK: Pearson; 2019. 655 p.

27. 10.1016/j.leaqua.2012.10.001 Stentz JE, Plano Clark VL, Matkin GS. Applying mixed methods to leadership research: A review of current practices. Leadersh Q [Internet]. 2012;23(6):1173-83. Available from: http://dx.doi.org/10.1016/j.leaqua.2012.10.001.

28. Wang $H$, Demerouti $E$, Bakker AB. A review of job crafting research: The role of leader behaviors in cultivating successful job crafters. In: Sharon K. Parker UKB, editor. Proactivity at Work: Making Things Happen in Organizations. 1st editio. New York: Routledge.; 2016. p. 77-104. 
29. Thun S, Bakker AB. Empowering leadership and job crafting: The role of employee optimism. Stress Heal [Internet]. 2018 Jun 8 [cited 2018 Sep 22]; Available from: http://www.ncbi.nlm.nih.gov/pubmed/29882309.

30. Sox HC, Higgins MC, Owens DK. Expected Value Decision Making. In: Medical Decision Making. John Wiley \& Sons, Ltd; 2013. p. 143-69.

31. Bates D, Mächler M, Bolker B, Walker S. Fitting Linear Mixed-Effects Models using Ime4. J Stat Softw [Internet]. 2015 Jun 23 [cited 2019 Dec 18];67(1):1-47. Available from: http://arxiv.org/abs/1406.5823.

32. Lipsey M, Wilson D. Practical meta-analysis (Applied Social Research Methods). Thousand Oaks: SAGE Publications; 2000.258 p.

33. Nakagawa S, Johnson PCD, Schielzeth $\mathrm{H}$. The coefficient of determination R2 and intra-class correlation coefficient from generalized linear mixed-effects models revisited and expanded. J R Soc Interface. 2017 Sep 1;14(134).

34. Nowell L, Norris J, White E, Moules D. N. Thematic Analysis: Striving to Meet the Trustworthiness Criteria. Vol. 16, International Journal of Qualitative. 2017.

35. http://doi.wiley.com/10.1111/medu.13124

Varpio L, Ajjawi R, Monrouxe LV, O'Brien BC, Rees CE. Shedding the cobra effect: problematising thematic emergence, triangulation, saturation and member checking. Med Educ [Internet]. 2017 Jan [cited 2019 Jul 16];51(1):40-50. Available from: http://doi.wiley.com/10.1111/medu.13124.

36. van Nes F, Abma T, Jonsson H, Deeg D. Language differences in qualitative research: is meaning lost in translation? Eur J Ageing [Internet]. 2010 Dec [cited 2018 Dec 13];7(4):313-6. Available from: http://www.ncbi.nlm.nih.gov/pubmed/21212820.

37. Arnold KA, Connelly CE, Walsh MM, Martin Ginis KA. Leadership styles, emotion regulation, and burnout. J Occup Health Psychol [Internet]. 2015 Oct [cited 2019 Apr 3];20(4):481-90. Available from: http://www.ncbi.nlm.nih.gov/pubmed/25844908.

38. Hildenbrand K, Sacramento CA, Binnewies C. Transformational leadership and burnout: The role of thriving and followers' openness to experience. J Occup Health Psychol [Internet]. 2018 Jan [cited 2019 Apr 3];23(1):31-43. Available from: http://doi.apa.org/getdoi.cfm?doi=10.1037/ocp0000051.

39. Appelbaum NP, Lee N, Amendola M, Dodson K, Kaplan B. Surgical Resident Burnout and Job Satisfaction: The Role of Workplace Climate and Perceived Support. J Surg Res [Internet]. 2019 Feb [cited 2019 Jun 28];234:20-5. Available from: https://linkinghub.elsevier.com/retrieve/pii/S002248041830605X.

40. Lases L, Arah OA, Busch ORC, Heineman MJ, Lombarts KMJMH. Learning climate positively influences residents' work-related wellbeing. Adv Heal Sci Educ [Internet]. 2019 May 5 [cited 2019 Jul 17];24(2):317-30. Available from: http://www.ncbi.nlm.nih.gov/pubmed/30519786.

41. Lee N, Appelbaum N, Amendola M, Dodson K, Kaplan B. Improving resident well-being and clinical learning environment through academic initiatives. J Surg Res [Internet]. 2017 Jul 1 [cited 2018 Sep 19];215:6-11. Available from: http://www.ncbi.nlm.nih.gov/pubmed/28688662.

42. Hu YY, Parker SH, Lipsitz SR, Arriaga AF, Peyre SE, Corso KA, et al. Surgeons' Leadership Styles and Team Behavior in the Operating Room. J Am Coll Surg [Internet]. 2016 Jan [cited 2018 Dec 22];222(1):41-51. Available from: http://www.ncbi.nlm.nih.gov/pubmed/26481409.

43. Horwitz IB, Horwitz SK, Daram P, Brandt ML, Brunicardi FC, Awad SS. Transformational, transactional, and passive-avoidant leadership characteristics of a surgical resident cohort: analysis using the multifactor leadership questionnaire and implications for improving surgical education curriculums. J Surg Res [Internet]. 2008 Jul [cited 2018 Sep 22];148(1):49-59. Available from: http://linkinghub.elsevier.com/retrieve/pii/S0022480408001911.

44. Choi SL, Goh CF, Adam MBH, Tan OK. Transformational leadership, empowerment, and job satisfaction: the mediating role of employee empowerment. Hum Resour Health [Internet]. 2016 Dec 1 [cited 2019 Apr 3];14(1):73. Available from: http://www.ncbi.nlm.nih.gov/pubmed/27903294.

45. 10.1007/s10459-012-9398-4

Stegeman JH, Schoten EJ, Terpstra OT. Knowing and acting in the clinical workplace: trainees' perspectives on modelling and feedback. Adv Health Sci Educ Theory Pract [Internet]. 2013 Oct 16 [cited 2019 Jul 17];18(4):597-615. Available from: http://link.springer.com/10.1007/s10459-012-9398-4.

46. Cope A, Bezemer J, Mavroveli S, Kneebone R. What Attitudes and Values Are Incorporated Into Self as Part of Professional Identity Construction When Becoming a Surgeon? Acad Med [Internet]. 2017 Apr [cited 2019 Jul 18];92(4):544-9. Available from: http://insights.ovid.com/crossref?an=00001888-201704000-00047. 
47. Nisar PJ, Scott HJ. Key attributes of a modern surgical trainer: Perspectives from consultants and trainees in the United Kingdom. J Surg Educ. 2011 May 1;68(3):202-8.

48. Kissane-Lee NA, Yule S, Pozner CN, Smink DS. Attending Surgeons' Leadership Style in the Operating Room: Comparing Junior Residents' Experiences and Preferences. J Surg Educ. 2016 Jan 1;73(1):40-4.

49. Thun S, Bakker AB. Empowering leadership and job crafting: The role of employee optimism. Stress Heal [Internet]. 2018 Oct [cited 2019 Apr 3];34(4):573-81. Available from: http://www.ncbi.nlm.nih.gov/pubmed/29882309.

50. Wang H, Demerouti E, Le Blanc P. Transformational leadership, adaptability, and job crafting: The moderating role of organizational identification. J Vocat Behav [Internet]. 2017 Jun 1 [cited 2018 Oct 3];100:185-95. Available from:

https://www.sciencedirect.com/science/article/pii/S0001879117300271.

51. Elshout R, Scherp E, van der Feltz-Cornelis CM. Understanding the link between leadership style, employee satisfaction, and absenteeism: a mixed methods design study in a mental health care institution. Neuropsychiatr Dis Treat [Internet]. 2013 Jun [cited 2019 Apr 4];9:823-37. Available from: http://www.dovepress.com/understanding-the-link-between-leadership-style-employeesatisfaction-peer-reviewed-article-NDT.

52. Green AE, Miller EA, Aarons GA. Transformational Leadership Moderates the Relationship Between Emotional Exhaustion and Turnover Intention Among Community Mental Health Providers. Community Ment Health J [Internet]. 2013 Aug 4 [cited 2019 Apr 3];49(4):373-9. Available from: http://www.ncbi.nlm.nih.gov/pubmed/22052429.

\section{Supplementary Files}

This is a list of supplementary files associated with this preprint. Click to download.

- Appendix1.docx 